(Aus dem physiologischen Laboratorium der zoologischen Station zu Neapel.)

\title{
Ueber \\ den Einfluss der Zerstörung des Labyrinthes beim Seepferdchen nebst einigen Bemerkungen über das Sehwimmen dieser Tiere.
}

Von

\section{Dr. Alfred Fröhlich (Wien).}

(Mit 1 Textfgur und Tafel I.)

Zur Ergänzung meiner in Bd. 102 und 103 dieses Archives ververöffentlichten Studien über die Erscheinungen nach Zerstörung der Statozysten verschiedener wirbelloser Tiere $^{1}$ ) sei im Folgenden in Kürze über einige Versuche ähnlichen Charakters am Seepferdehen berichtet, die im April 1903 an der zoologischen Station zu Neapel ausgeführt wurden.

Als Versuchsobjekt dienten verschiedene Arten des Seepferdchens, meist Exemplare von Hippocampus brevirostris, mitunter auch von H. guttulatus.

Wie bekannt, erfolgt die Fortbewegung des Seepferdchens stets in vertikaler Orientirung des Tieres; senkrecht im Wasser schwebend bewegt es sich in langsamer, graziöser Weise durch das undulirende Spiel der insektenflügelartigen paarigen Brustflossen und der unpaarigen Rückenflosse. Auf- und Niedertauchen erfolgt gleichfalls, ohne dass die vertikale Orientirung aufgegeben wird.

Es handelte sich darum, zu untersuchen, ob diese Haltung durch Zerstörung des Labyrinthes ${ }^{2}$ ) aufgehoben resp. geändert wird.

1) A. Fröhlich, Studien über die Statozysten. I. Mitteilung. Versuche an Zephalopoden und Finschlägiges aus der menschlichen Pathologie. Arch. $f$. d. ges. Physiol. Bd. 102 S. 415-472. 1904, und A. Fröhlich, Studien über die Statozysten wirbelloser Tiere. II. Mitteilung. Versuche an Krebsen. Arch. f. d. ges. Physiol. Bd. 103 S. 149-168. 1904.

2) Das häutige Labyrinth der Teleostier ist von der Schädelböhle nur durch die Dura mater getrennt; ein eigentliches knöchernes Labyrinth besteht nicht. 
Ueber den Einfl. d. Zerstörung des Labyrinthes beim Seepferdehe, etc. 85

In Anbetracht der fast mikroskopischen Verhältnisse (die grösste Länge des membranösen Labyrinthes beträgt unter Zugrundelegung der Retzius'schen Abbildung nur 2,5 mm) und der so delikaten Struktur konnte an eine glatte Exstirpation dieses Organes nicht gedacht werden. Ich musste mich daher darauf beschränken, mit einem elektrisch betriebenen rotirenden Bohrer von ungefähr $2 \mathrm{~mm}$ Durchmesser die betreffende Gegend nach Art der zahnärztlichen Technik auszubohren und die gesetzte Läsion durch nachherige mikroskopische Untersuchung festzustellen. Die Ausbohrung wurde nie so lange fortgesetzt, bis das Gehirn blosslag; der bei Berührung des Labyrinthes eintretende Nystagmus gab einen wertvollen Index $a b$.

Die Tierchen wurden während des nur kurze Zeit dauernden Eingriffes durch ein in das Maul eingeführtes und mit Seewasser gespeistes Glasröhrchen künstlich respirirt und nach Vollendung der Operation sofort ins Aquarium zurückversetzt, woselbst sie regelmässig durch eine Reihe von Tagen, manchmal über eine Woche am Leben erhalten werden konnten.

Die Bewegungsstörungen traten im unmittelbaren Anschlusse an die Operation in Erscheinung und gingen während der ganzen weiteren Lebensdauer nicht mehr zurück. Im Vordergrunde stehen bei jedem Schwimmversuche (Vorwärtsbewegung) eintretende Rotationen um die Längsachse, und zwar (von oben gesehen) bei Operation links im Sinne eines Uhrzeigers, bei Operation rechts gegen die Richtung eines Uhrzeigers.

Da ein Seepferdchen, dem man die rechte Brustflosse und die Rückenflosse abgeschnitten hat, das also nur mehr die linke Brustflosse besitzt, sich (bei intakten Labyrinthen) von oben gesehen im Sinne des Uhrzeigers dreht, so lann gefolgert werden, dass durch die einseitige Labyrinthzerstörung eine Störung in der Arbeit der der Seite der Operation gegenüberliegenden Flosse gesetzt wurde, die unter Berücksichtigung der analogen Erfahrungen an den meisten anderen Tierklassen nicht anders denn als Schwächung der Muskulatur der betreffenden (kontralateralen) Flosse aufgefasst werden darf entsprechend der $\mathrm{E}$ wald'schen Lehre vom Tonuslabyrinth.

Ferner erfolgen beim einseitig labyrinthlosen Tiere Manègebewegungen (Kreisen), und zwar in gleichem Sinne wie die Rotationen um die Körperlängsachse.

Bei all diesen abnorme'n Bewegungen wird die normale, d. i. vertikale Stellung im Wasser nicht verlassen; vielmehr wird sowohl 
nach einseitiger wie nach doppelseitiger Labyrintbzerstörung das Seepferdchen in der Ruhe stets, beim Schwimmen fast immer mit Ausnahme einiger im Nachfolgenden zu besprechender Fälle in senkrechter Stellung angetroffen.

Eine regelmässige Folge der einseitigen Labyrinthzerstörung ist eine veränderte Haltung der einzelnen Körperabschnitte zu einander. Der Kopf wird meist gegen die Seite der Verletzung bin geneigt gehalten, der Schwanz oft nach der operirten Seite hin flektirt. Oft wird der Kopf stark nach hinten gestreckt gehalten, so dass Kopf und Leib fast in eine Gerade zu liegen kommen; zugleich wird der Kopf nach der Seite der Operation geneigt (Beobachtung 5). Der Schwanz wurde in diesem Falle nach der gesunden linken Seite gebeugt, wie um die durch die anormale Stellung des Kopfes bedingte Verlegung des Schwerpunktes auszugleichen. In anderen Fällen lag die Schnauze der vorderen Leibeswand an. Manchmal wurde der Schwanz in gestreckter Haltung ventralwärts flektirt und in einem annähernd rechten Winkel zum Leibe steif nach vorne gestreckt gehalten: aus dieser Stellung erfolgte das Schwimmen in Horizontalstellung des Rumpfes, Kopf voraus (siehe Tafel Beobachtung 5). Es kamen auch eigenartige Manègebewegungen in diesem Falle in der Weise zu Stande, dass das Tier beim Schwimmen auf der rechten (operirten) Seite fast horizontal, mit nur geringer Neigung (schätzungsweise $10 \%$ ) im Wasser lag und sich in Kreisen von 10-20 cm Durchmesser umherbewegte. Ueberpurzeln kam in keinem Falle zur Beobachtung. In Ruhe und bei nicht zu stürmischer Bewegung blieb die vertikale Orientirung im Wasser stets annähernd erhalten; bei aufgeregtem Schwimmen entstanden durch die oben beschriebene anormale Kopf-, Körper- und Schwanzhaltung eigenartige Schraubenbewegungen.

Die Schwimmstörungen der übrigen operirten Fälle entsprachen der hier ausführlich mitgeteilten Beobachtung 5, so dass von einer Wiedergabe der übrigen Protokolle abgesehen werden kann. Die Abbildungen auf der Tafel geben ein Bild der oftmals ganz absonderlichen Haltungen und Stellungen der operirten Tiere während des Schwimmens.

Nach Ablauf der Beobachtungszeit, die sich in der Regel auf eine Woche erstreckte, wurden die Tiere dekapitirt, die Köpfe in Formol fixirt und sodann nach vorausgegangener Entkalkung in Zelloidin eingebettet und in Serien zerlegt, um den Grad der Ver- 
Ueber den Einfl. d. Zerstörung des Labyrinthes beim Seepferdchen etc. 87

letzung durch mikroskopische Untersuchung kontroliren zu können. Im Folgenden seien die histologischen Befunde, bei deren Erhebung mir mein Freund Dozent Dr. Grosser behilflich war, wofür ich ihm hier bestens danke, in Kürze mitgeteilt.

Beobachtung 1. Rechts operirt.

Mittelhirn und Nachhirn verletzt und auf die Seite gedrängt; das Gehör organ fehlt rechts vollständig.

Links: Gehörorgan intakt.

Beobachtung 2. Rechts operirt.

Gehirn unverletzt. Vorhanden sind rechts: die zwei vorderen Ampullen. Noch in der Gegend der beiden Ampullen ist das membranöse Labyrinth eröffnet. Gerinnselbildung im endolymphatischen Apparate in der Form von feinkörnigen Niederschlägen.

Links: Gehörorgan normal.

Beobachtung 3. Links operirt.

Das Mittelhirn teilweise oberflächlich lädirt, mit Blut und Exsudat bedeckt. Das Gehirn ist ala Ganzes nach der rechten Seite hin gedrängt und daher im Schnitt asymmetrisch gelagert.

Vom Gehörorgan ist links überhaupt nichts mehr zu sehen.

Rechts: Gehörorgan gut erhalten.

Beobachtung 4. Rechts operirt.

Das Gehirn ein ganz klein wenig auf die Seite gedrängt, im Wesentlichen unverletzt.

Das rechte Gehörorgan ist eröffnet und kollabirt; es erscheinen die vordere und äussere Ampulle verletzt und gleichzeitig aus ihrer Lage gebracht.

Das linke Gehörorgan ganz unverletzt.

Beobachtung 5. Rechts operirt.

Das Gehirn auf die Seite gedrängt; es sieht aus, als ob es nach vorne gequetscht worden wäre.

Das rechte Gehörorgan fehlt vollständig.

Das Gehörorgan der linken, unverletzten Seite ist gut; es erscheint mit feinstem Gerinnsel erfüllt.

Beobachtung 6. Links operirt.

Gehirn mitverletzt, und zwar hauptsächlich das Mittelhirn; auch das Zwischenhirn ist stark nach der rechten Seite verdrängt und auch lädirt.

Gehörorgan links radikal exstirpirt.

Rechts: Gehörorgan gut erhalten.

Beobachtung 7. Rechts operirt.

Gehirn bloss etwas verschoben und im Mittelhirnbereiche etwas gedrückt. Gehörorgan fehlt rechts vollständig.

Links: Gehörorgan kollabirt. Sonst unverletzt. 
Aus diesen Befunden geht hervor, dass es in einigen Fällen unzweifelhaft gelungen ist, das Gehörorgan isolirt ohne Läsion des Gehirnes zu zerstören, so dass die beobachteten Anomalien in Bewegung und Haltung nur auf die Läsion des Labyrinthes bezogen werden können. Auch in den übrigen Fällen (mit Ausnahme von Beobachtung 6), in denen das Gehirn bei der Ausbohrung beschädigt wurde, war die Gehirnverletzung nur unwesentlich, so dass auch in diesen Fällen bei der Gleichartigkeit der Störungen mit den bei isolirter Labyrinthzerstörung beobachteten diese nicht auf die Verletzung des Zentralnervensystems bezogen werden dürfen.

II.

In ganz analoger Weise, wie dies in früheren Publikationen (s. d. Archiv Bd. 102 u. 103) für Zephalopoden und Krustazeen von mir beschrieben wurde, zeigten sich auch beim Seepferdchen nach der Zerstörung des Labyrinthes gewisse Reflexe gesteigert. Es genügte bei vielen Individuen, die zu diesem Zwecke aus dem Wasser genommen und flach auf den Tisch gelegt wurden, den Schwanz seitlich ganz sanft mit einer Bleistiftspitze oder einem ähnlichen Gegenstande zu berühren, um eine brüske seitliche Flexion des Sehwanzes gegen den Rumpf hervorzurufen, so energisch, dass die Schwanzspitze den Kopf berübrte. Besonders ausgesprochen war dieses Verhalten bei Berührung auf der Seite der Verletzung. Bei einem normalen Seepferdchen erfolgt selbst auf kräftige Berührung des Schwanzes nur eine schwache Seitwärtsbewegung dieses Körperteils.

Mitunter treten während des Schwimmens labyrinthoperirter Seepferdchen heftig pendelnde Schwanzschläge in seitlicher Richtung (nach rechts und links) auf, die ich beim normalen Tiere niemals beobachten konnte.

Im Ansehlusse an die Operation war oft schon wenige Minuten nachher eine - selbst stundenlang andauernde - Rastlosigkeit des Tierchens sichtbar. Es schwamm sodann unausgesetzt unter steten Drehungen um die Körperlängsachse umher und refüsierte das Anklammern mit dem Schwanze an dargebotene feste Objekte, an die sich ein normales Seepferdchen sofort anklammert, um sodann in Ruhe zu verharren. 


\section{III.}

Aus dem Mitgeteilten geht hervor, dass die normale vertikale Orientirung des Seepferdchens im Wasser durch Labyrinthzerstörung zu allermeist nicht beeinflusst wird. Einzelner Ausnahmen wurde oben auf Seite 86 gedacht. In Anbetracht des Umstandes, dass selbst ein totes - sei es labyrinthoperirtes, sei es normales Seepferdchen die Vertikalstellung im Wasser nicht aufgibt, können die geschilderten Verhältnisse wohl nur durch eine besondere statische Anordnung der Organe des Körpers bedingt sein. Eine solche liegt denn auch in der Lage der Schwimmblase vor. Wie ein Blick auf beistehende Abbildung eines Seepferdchens, dem die linke Rumpfwand wegpräparirt wurde, lehrt, ist beim Seepferdchen dieSchwimmblase $(S)$ so stark gegen den Kopf zu verschoben, dass der Schwerpunkt kaudal von derselben liegen muss. Das Gewicht des langen Schwanzes, der beim Schwimmen fast gestreckt gehalten wird, dient dazu, um den Schwerpunkt noch tiefer zu verlegen. Durch diese beiden Momente, die dem Kopfe unmittelbar benachbarte Lage der Schwimmblase und das Gewicht des Schwanzes, wird die Senkrechtstellung des Körpers im Wasser herbeigeführt und auch erklärt, dass diese vertikale Orientirung stets, auch im Tode, eingehalten wird. Dass diese statischen Verhältnisse sehr leicht geändert werden können, davon kann man sich durch einen einfachen Versuch überzeugen. Injizirt man einem lebenden oder toten Seepferdehen mit einer Pravaz'schen Spritze einige Tropfen Wasser in die Schwimmblase, so ist die vertikale Orientirung aufgehoben, und man kann ein solches Tierchen horizontal auf den Boden des Aquariums hinlegen. Injizirt man hingegen einem toten Seepferdchen (das schliess- 
90 Alfred Fröhlich: Ueber den Einfl. d. Zerstörung d. Labyrinthes etc.

lich nach einigen Tagen horizontal auf dem Aquariumboden daliegt) eine gleich grosse Menge Luft in die Schwimmblase oder in die Leibeshöhle, so bleibt es fortan senkrecht im Wasser stehen. Die Verhältnisse liegen demnach ganz ähnlich wie bei dem Pneumatophor der Siphonophoren, der gleichfalls rein mechanisch dem Siphonophorenstocke eine vertikale Haltung im Wasser verleiht.

Für die Ueberlassung des Arbeitsplatzes an der zoologischen Station zu Neapel spreche ich dem hohen k. k. Ministerium für Kultus und Unterricht meinen ergebensten Dank aus. 

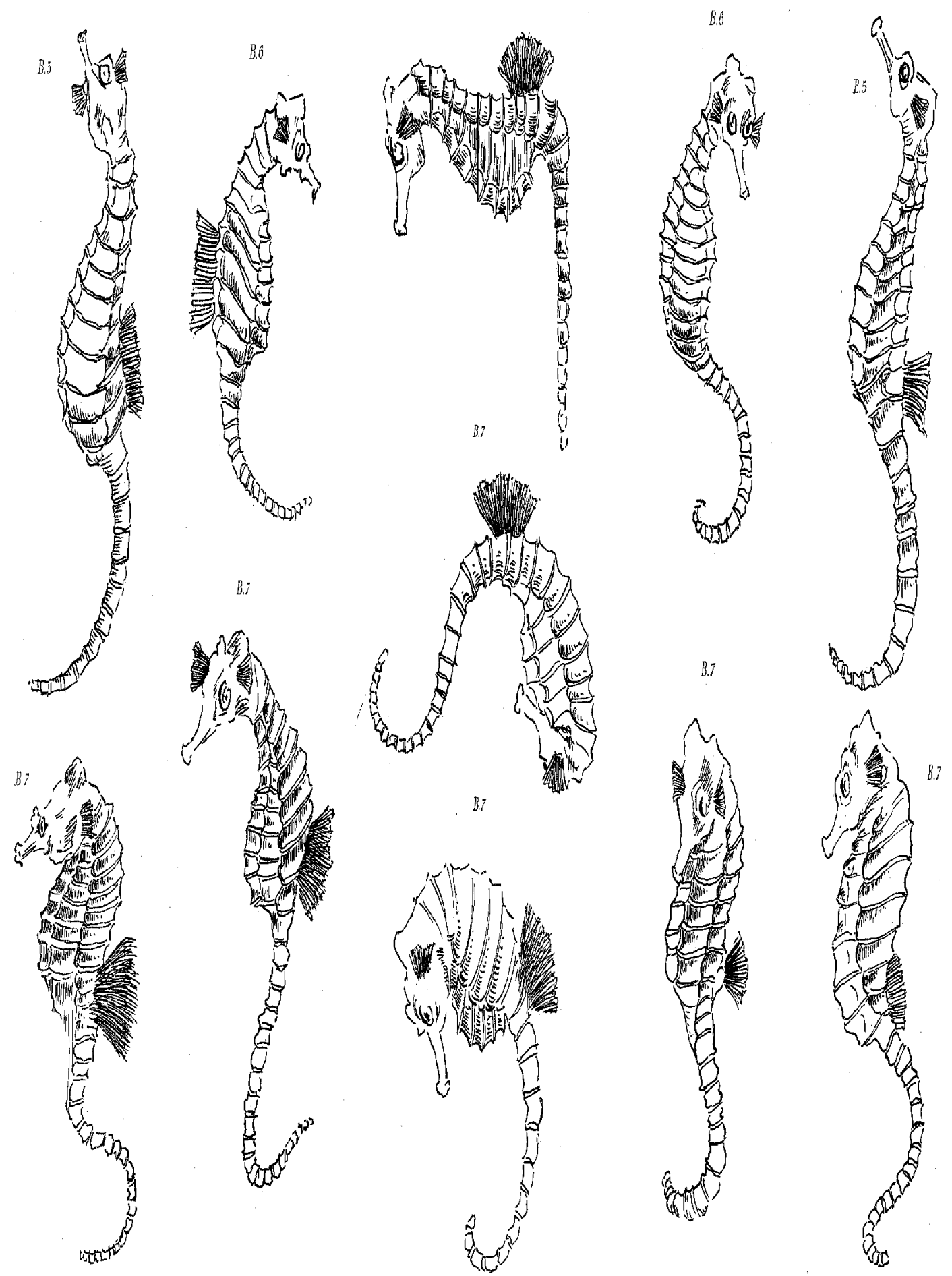